\title{
Postburn Elevation in Fibrin Degradation Product is Related to Burn Severity
}

Prasun Barua ${ }^{1 *}$

Mohammed Kaisar lqbal ${ }^{2}$

Mahmudul Haque ${ }^{3}$

'Department of Biochemistry

Army Medical College

Chattogram, Bangladesh.

${ }^{2}$ Ukhiya Upazila Health Complex

Cox's Bazar, Bangladesh.

${ }^{3}$ Department of Biochemistry Chattogram Medical College

Chattogram, Bangladesh.

\begin{abstract}
Background: Burn is reported to cause significant alteration of haemocoagulative parameters, but due to lack of proper understanding of burn coagulopathy, only the routine coagulation tests are generally used, even though these provide limited diagnostic or prognostic information about burns and postburn complications. So, this study specifically aimed to characterize the changes in plasma Fibrin Degradation Product (FDP) together with routine coagulative parameters and their relations with burn-severity.
\end{abstract}

Materials and methods: This cross-sectional comparative study was carried out in the Department of Biochemistry and Department of Burn and Plastic Surgery of Chattogram Medical College Hospital from January to December in 2014. 130 subjects of 18 - 65 years enrolled in two groups by nonprobability consecutive sampling. Group-A consisted of 80 patients with minor, moderate and major burns as per American Burn Association severity classification, whereas Group-B included 50 age and sex-matched healthy subjects. Plasma FDP was estimated by immunoturbidimetric method in STA compact automated analyser. Bleeding Time (BT), Clotting Time (CT) and Prothrombin Time (PT) were also determined using standard methods.

Results: Burn patients had significantly higher mean FDP $(10.13 \pm 5.63 \mu \mathrm{g} / \mathrm{ml})$ than controls $(2.50 \pm 1.56 \mu \mathrm{g} / \mathrm{ml})$ which increased with increase in burn-severity (4.83, 8.12 and $15.48 \mu \mathrm{g} / \mathrm{ml}$ in minor, moderate and major burns respectively). Plasma FDP increased in $91.25 \%$ burn patients. While BT and CT were mostly unchanged, PT was prolonged in $66.25 \%$ burn patients (Mean $=15$ seconds) but that did not seem to correlate with burn-severity or with FDP.

Conclusion: Plasma FDP increased significantly in burn patients showing better and consistent correlation with burn-severity than routine coagulation parameters.

Key words: Burn; Coagulation; Fibrinolysis, Fibrin Degradation Product (FDP); Bleeding Time (BT); Clotting Time (CT); Prothrombin Time (PT).

\section{INTRODUCTION}

Burn is one of the leading causes of morbidity and mortality, especially in the low and middle-income countries ${ }^{1}$. Burn injury is traditionally referred to as a common initiating factor of acute coagulopathy ${ }^{2-5}$. Burn-induced coagulopathy and its severity often determine patient-outcome $e^{6,7}$. It also increases the risk of several complications like Disseminated Intravascular coagulation (DIC), thromboembolism, multi-organ failure and postburn sepsis ${ }^{6,8-11}$. Although burn is reported to induce dynamic and variable changes in both coagulation (Hypo/hypercoagulation) and fibrinolytic (Hypo/hyperfibrinolysis) systems, routine coagulation tests, comprising platelet count, Bleeding Time (BT), Clotting Time (CT) and Prothrombin 
Time (PT) etc. provide very limited insights into this pathology ${ }^{4-6,11-14}$. Fibrin degradation product (FDP) included in the current diagnostic criteria of DIC, holds the unique promise of offering a better understanding of burn cases as it not only indicates active fibrinolysis but also generally describes the state of coagulation or fibrin formation. While earlier FDP studies in burn patients were biased largely by low-sensitivity assays and restricted parameters, most of the later studies are affected by limited number of cases and heterogenicity of burn population $^{3-4,6,11-17}$. Thus, regardless of some encouraging advancements, postburn modifications in coagulation and fibrinolytic systems are still inadequately understood, leaving tantalizing gaps in burn literature. This also limits the potential diagnostic, prognostic and therapeutic utilities of FDP assessment in burn and postburn complications. So, this study was undertaken to analyse the changes in plasma FDP in burn patients compared to healthy individuals and to explore its relations with burn-severity and routine coagulation parameters.

\section{MATERIALS AND METHODS}

This cross-sectional comparative study was carried out in the Department of Biochemistry and Department of Burn and Plastic Surgery of Chattogram Medical College Hospital from January to December in 2014. Proper permission was taken from concerned departments and the Ethical Review Committee of Chattogram Medical College Hospital. All participants gave informed written consent. 130 subjects enrolled in two groups by nonprobability consecutive sampling were studied. Group-A consisted of 80 burn patients (14 minor, 38 moderate and 28 major burns as per American Burn Association severity classification). Group-B included 50 age and sex-matched healthy subjects. The inclusion and exclusion criteria were as follows:

Group A: Inclusion criteria:

Burn patients of $18-65$ years admitted in the Burn and Plastic Surgery Department of Chattogram Medical College Hospital.

\section{Exclusion criteria:}

Patients with hepatic or renal disease, cancer, pre-existing coagulation disorders including DIC, Deep Vein Thrombosis (DVT) sepsis, pregnancy, history of recent blood transfusion, smoking, patients taking drugs like barbiturate, heparin, aspirin, streptokinase, urokinase, oral contraceptive pill, etc that may affect coagulation or fibrinolytic system.

\section{Group B: Inclusion criteria:}

Healthy individuals from community aged 18-65 years.

\section{Exclusion criteria:}

Subjects known or suspected to have any disease or pathology, pregnancy, history of recent blood transfusion, smoking on drugs that may affect coagulation or fibrinolytic system.

Early morning blood samples were taken during the second week after burn when FDP concentration is expected to be at its peak. Plasma FDP was estimated by immuno-turbidimetric method in STA compact automated analyser. BT, CT and PT were also determined using standard methods. Data were processed and analysed with computer-based statistical software. Quantitative data were expressed as mean \pm SD (Standard Deviation) and qualitative in percentage. The confidence level was fixed at $95 \%$ and a "p" value of 0.05 or less was considered significant. Different tests of statistical significance were done as appropriate.

\section{RESULTS}

Table I: Distribution of the study groups $(n=130)$

$\begin{array}{lcc}\text { Study Groups } & \text { Frequency (n) } & \text { Percentage (\%) } \\ \text { Group A (Burn Patients) } & 80 & 61.53 \\ \text { Group B (Controls) } & 50 & 38.47 \\ \text { Total } & 130 & 100.0\end{array}$

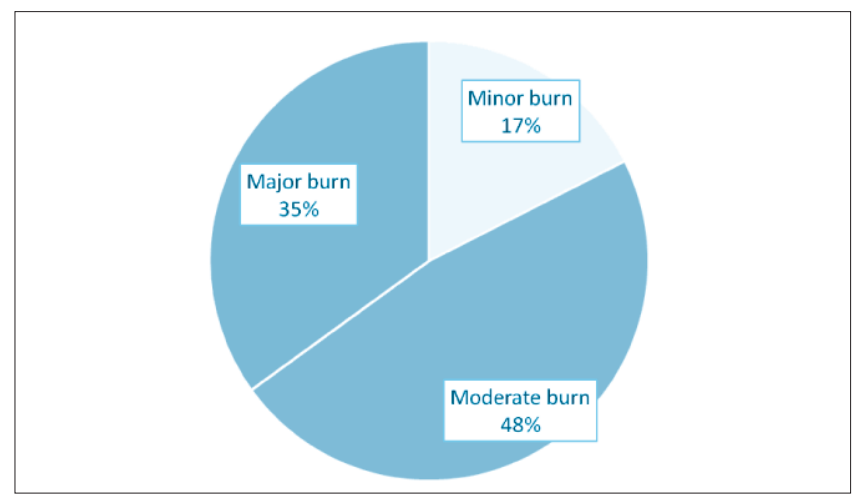

Figure 1 : Pie chart showing distribution of burn patients according to severity, $\mathrm{n}=80$

Table II : Comparison of plasma FDP between Group A and Group B

\begin{tabular}{lllllll} 
& Groups & n & Mean & \pm SD & Range & p value \\
FDP & Group A & 80 & 10.13 & \pm 5.63 & $3.83-33.00$ & $<0.0001$ \\
$(\mu \mathrm{g} / \mathrm{ml})$ & Group B & 50 & 2.50 & \pm 1.56 & $0.40-5.30$ & \\
\hline
\end{tabular}

Mean plasma FDP was significantly higher in burn patients than in controls. Reference [FDP]: $<5 \mu \mathrm{g} / \mathrm{ml}$.

Table III: Comparison of FDP amongst burn patients of different severity (With ANOVA test of significance)

\begin{tabular}{cllllll} 
& Burn severity & $\mathbf{n}$ & Mean & \pm SD & Range & p value \\
FDP & Minor & 14 & 4.83 & \pm 0.79 & $3.83-6.10$ & \\
$\mu \mathrm{g} / \mathrm{ml}$ & Moderate & 38 & 8.12 & \pm 1.14 & $6.33-10.30$ & \\
& Major & 28 & 15.48 & \pm 6.40 & $8.70-33.00$ & $<0.0001$ \\
& Total & 80 & 10.13 & \pm 5.63 & $3.83-33.00$ & \\
\hline
\end{tabular}

Mean concentration of FDP increased with increasing burn severity. 


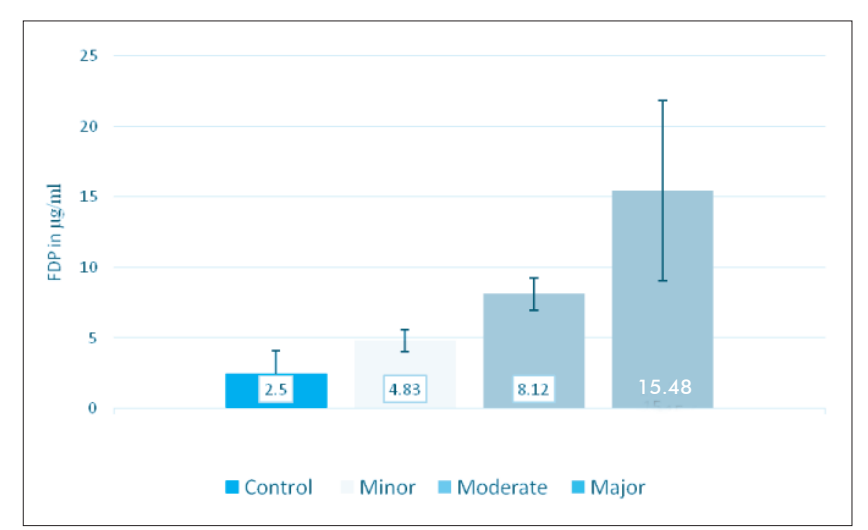

Figure 2 : Bar diagram showing mean $\pm \mathrm{SD}$ of plasma FDP in controls and burn patients

There was tendency of increase in FDP as the severity of burn increased.

Table IV : Association between burn and elevated FDP, $\mathrm{n}=$ 130 (With $\chi^{2}$ test of significance and odds ratio)

$\begin{array}{llll}\text { Groups } & \text { FDP increased } & \text { FDP normal } & \text { Total } \\ \text { Group A } & 73(91.25 \%) & 07(8.75 \%) & 80(100.00 \%) \\ \text { Group B } & 05(10.00 \%) & 45(90.00 \%) & 50(100.00 \%)\end{array}$

Comparison of burn patients with controls using Chi-squared tests and odds ratio proved strong and statistically significant association between burn and elevated FDP.

Table V : Comparison of PT amongst burn patients of different severity (with ANOVA test significance) $(n=80)$

\begin{tabular}{lllllll}
\multicolumn{2}{l}{ Burn severity } & $\mathbf{n}$ & Mean & \pm SD & Range & p value \\
PT & Minor & 14 & 14.36 & \pm 1.39 & $12-16$ & $>0.05$ \\
& Moderate & 38 & 14.95 & \pm 0.99 & $13-17$ & \\
& Major & 28 & 15.39 & \pm 1.66 & $13-19$ & \\
& Total & 80 & 15.00 & \pm 1.36 & $12-19$ & \\
\hline
\end{tabular}

PT was prolonged in $66.25 \%$ of burn patients but the prolongation was not analogous to burn severity. Reference PT: 12-14 seconds.

\section{DISCUSSION}

Our study emphasizes on characterization of FDP using latest available immunoassay technique together with routine coagulative parameters (BT, CT, PT) in eighty (80) carefully selected burn patients of different severity. All the tests were done during second postburn week. As shown in results the concentrations of FDP were significantly higher in burn patients $(10.13 \pm 5.63 \mu \mathrm{g} / \mathrm{ml})$ than in healthy subjects $(2.50 \pm$ $1.56 \mu \mathrm{g} / \mathrm{ml})$. Rise in mean FDP was also proportionate to burn severity $(4.83 \mu \mathrm{g} / \mathrm{ml}, 8.12 \mu \mathrm{g} / \mathrm{ml}$ and $15.48 \mu \mathrm{g} / \mathrm{ml}$ respectively in mild, moderate and major burns). These findings while contrast with some of the earlier studies are in line with the more recent ones. Meyers and Milwaukee analysed FDP in 77 burn patients using red cell hemagglutination inhibition immuno-assay to find only a modest elevation in the severely burned group that did not show any correlation with burn size or morbidity ${ }^{3}$. Bartlett et al also reported very similar results. However, Eurenius et al employing staphylococcal clumping technique noticed significant elevation of FDP well beyond 10 days after burn $(n=35)^{5}$. Curreri et al also reported grossly elevated FDP during the first two post-burn weeks where only $7 \%$ of the 55 burn patients had minimal elevation of FDP (Mean $=6.77 \pm 0.07 \mu \mathrm{g} / \mathrm{ml}$ ) and $93 \%$ had at least four times maximum normal concentration $(\text { Mean }=41.33 \pm 4.7 \mu \mathrm{g} / \mathrm{ml})^{15}$. Caprini et al too gave a comparable result ${ }^{4}$. Other researchers used D-dimer (A type of FDP) to better appreciate the postburn coagulopathy and observed significant rise in D-dimer level often persisting for weeks. They concluded that both thrombotic and fibrinolytic pathways are directly triggered proportionally to the extent of burn injury $6,11-14,17$. Rise in FDP was detectable as early as day as on day 1 and usually reached a peak during the second postburn week showing compatibility with our results ${ }^{4,6,11,16}$.

Unlike that in FDP, postburn changes in clotting factors and platelets are more variable. Immediately after burn there is a brief period of hypercoagulability that prevents blood loss. Then ensues a state of hypocoagulability due to progressive thrombocytopenia and decrease in clotting factors for the first 3 days, with greater change occurring with increasing extent of burn. Patient may have bleeding tendency, BT, CT etc. may be prolonged during this period. Hence, in patients with major burns, required operations should be carried out during the first 24 hours after injury or delayed until the platelet count has reached its nadir and is rising. Platelet count gradually returns to normal, reaching baseline values by 1 week and rebounding to abnormally high levels during the remainder of the acute phase ensuring a second hypercoagulable state. So, in the second postburn week when we estimated the BT and CT of our patients, they were probably in the phase of coagulative rebound resulting in normal values of these parameters without any sign of bleeding $g^{4-6,11-13}$. So, coagulation is a rapid and dynamic process whose abnormalities may be short-lived and missed by BT and CT.

In agreement with other burn studies, PT was prolonged in $66.25 \%$ of our patients, but the prolongation was not analogous to burn severity or to changes in FDP. This validates some previous claims that PT is not sensitive enough to detect the subtle alterations in coagulation and fibrinolytic systems in burn patients ${ }^{12,14,18}$. Moreover, majority of thermally injured patients do not always present with prolongation of PT i.e in the early post-burn period, PT may be normal despite marked changes in specific coagulation and fibrinolytic markers ${ }^{4-5,18}$. Studies report time course-related changes in coagulative and fibrinolytic markers, with most results normalizing between five and seven days unless complications develop $p^{4,6,11-16}$. The most serious of the complications are DIC and resultant multiorgan failure; triggering factors of which are sepsis or severe infection, endothelial injury, release of tissue factor and inflammatory cytokines ${ }^{9}$. Surgery, blood loss, hypovolaemia or 
excessive volume expansion, hypothermia and acidosis further aggravate the situation ${ }^{6,11,14}$. The incidence of DIC in burn differs depending on the diagnostic criteria and definitions used in each study. Nevertheless, the current scientific evidence is consistent with the hypothesis that biochemical changes suggestive for subclinical DIC (Hypercoagulability, hypo/hyperfibrinolysis) are commonplace in patients with burn trauma, and their severity increases exponentially with the severity of injury. Overt DIC seems to occur especially in critically ill burn patients or in those with severe burns (Up to third degree) and large involvement of body surface area ${ }^{9}$. DVT and $\mathrm{PE}$ are also now increasingly recognized as frequent complications in hospitalized burn patients ${ }^{19,20}$.

\section{CONCLUSION}

In this study, we observed significant elevation of FDP proportional to burn severity during the second postburn week when the routine coagulation markers (BT, CT, PT) were of little value. So, based on our findings and that of relevant literature we would like to conclude that FDP better characterizes burn induced coagulopathy and should be included in postburn coagulation assessment to determine injury-severity, hypercoagulability and risk of DIC or thromboembolic conditions.

\section{RECOMMENDATION}

Prospective multicentre study with close monitoring of all coagulation parameters (including FDP) is necessary to reveal the full picture of burn induced coagulopathy.

\section{ACKNOWLEDGEMENT}

We express our heartiest gratitude to the patients and staffs of Burn and Plastic Surgery Department of Chattogram Medical College Hospital for their participation and cooperation.

\section{DISCLOSURE}

All the authors declared no competing interest.

\section{REFERENCES}

1. Burns [Internet]. World Health Organization. World Health Organization. 2018 [Cited 2019 Jul 15]. Available from: https://www.who.int/en/news-room/fact-sheets/detail/burns.

2. Curreri PW et al. Coagulation abnormalities in the thermally injured patient. Current Topics Surg. 1970;2:401.

3. Meyers A. Fibrin split products in the severely burned patient. Arch. Surg. 1972;105:404.

4. Caprini JA, Lipp V, Zuckerman L et al. Hematologic changes following burns. J Surg Res. 1977;22(6):626-635.

5. Bartlett RH, Fong SW, Marrujo G, Hardeman J, Anderson W. Coagulation and platelet changes after thermal injury in man. Burns. 1981;7:370-377.

6. Garc a-Avello A, Lorente J, Cesar-Perez J et al. Degree of hypercoagulability and hyperfibrinolysis is related to organ failure and prognosis after burn trauma. Thromb Res.1998;89:59-64.

7. Niles SE, McLaughlin DF, Perkins JG, Wade CE, Li Y, Spinella PC, Holcomb JB. Increased mortality associated with the early coagulopathy of trauma in combat casualties. J Trauma. 2008;64:1459-1463.

8. Sevitt S. A review of the complications of burns, their origin and importance for illness and death. J Trauma. 1979;19(5):358-369.

9. Lippi G, Ippolito L, Cervellin G. Disseminated intravascular coagulation in burn injury. Semin Thromb Hemost. 2010;36(4):429-436.

10. Faucher LD, Conlon KM. Practice guidelines for deep venous thrombosis prophylaxis in burns. J Burn Care Res. 2007;28(5):661-663.

11. Lavrentieva A, Kontakiotis T, Bitzani M et al. Early coagulation disorders after severe burn injury: Impact on mortality. Intensive Care Med. 2008;34(4):700-706.

12. King DR, Namias N, Andrews DM. Coagulation abnormalities following thermal injury. Blood Coagul Fibrinolysis. 2010;21:666-669.

13. Van Haren RM, Thorson CM, Valle EJ, et al. Hypercoagulability after burn injury. J Trauma. 2013;75:37-43.

14. Kowal-Vern A, Gamelli RL, Walenga JM, Hoppensteadt D, Sharp-Pucci M, Schumacher HR. The effect of burn wound size on hemostasis: A correlation of the hemostatic changes to the clinical state. J Trauma. 1992;33:50-56.

15. Curreri PW, Wilterdink ME, Baxter CR. Characterization of elevated fibrin split products following thermal injury. Ann Surg. 1975;181(2):157-160.

16. Eurenius K, Rossi TD, McEuen DD, Arnold J, McManus WF. Blood coagulation in burn injury. Proc Soc Exp Biol Med.1974;147:878-882.

17. Tejiram S, et al. In-depth analysis of clotting dynamics in burn patients. J Surg Res. 2016;202:341-351.

18. Park MS, Martini WZ, Dubick MA, et al. Thromboelastography as a better indicator of postinjury hypercoagulable state than prothrombin time or activated partial thromboplastin time. J Trauma. 2009;67:266.

19. Wahl WL, Brandt MM. Potential risk factors for deep venous thrombosis in burn patients. J Burn Care Rehabil. 2001;22:128-131.

20. Harrington DT, Mozingo DW, Cancio L, Bird P, Jordan B, Goodwin CW. Thermally injured patients are at significant risk for thromboembolic complications. J Trauma. 2001;50:495-500. 